\title{
Surface complexation modeling of arsenic mobilization from goethite: Interpretation of in-situ experiments in a sedimentary basin of Inner Mongolia, China
}

\author{
L. Stolze ${ }^{1}$, D. Zhang ${ }^{2}$, H.M. Guo ${ }^{2} \&$ M. Rolle ${ }^{1}$ \\ ${ }^{1}$ Department of Environmental Engineering, Technical University of Denmark, Lyngby, Denmark \\ ${ }^{2}$ School of Water Resources and Environment, China University of Geosciences, Beijing, P.R. China
}

\begin{abstract}
We present and compare conceptual and numerical modeling approaches for a quantitative interpretation of in-situ experiments that consisted in monitoring the temporal change of adsorbed-As concentration by incubating As-loaded goethite coated sand in the groundwater. We employed the diffuse double layer (DDL) and charge distribution multisite complexation (CD-MUSIC) models to simulate the measured As-sorbed concentration and to investigate the effects of multicomponent adsorption processes on As mobility.
\end{abstract}

\section{INTRODUCTION}

Sorption competition and mineral-oxides reductive transformation have been recognized as predominant mechanisms controlling the release and mobility of arsenic in Asia (e.g., Neidhart et al., 2014). Despite the considerable effort toward understanding the geochemical mechanisms controlling the occurrence of arsenic, numerical modeling of these processes remain rare and mostly limited to well-controlled laboratory experiments (Rawson et al., 2016).

Surface complexation models (SCMs) have been widely applied as a powerful alternative to traditional empirical sorption modeling approaches (Dzombak \& Morel, 1990; Hiemstra \& Van Riemsdijk, 1996). However, these models are typically developed and/or applied to simple aqueous systems rather than complex environmental groundwater conditions.

Field experiments were recently performed in an As contaminated basin in Inner Mongolia, China (Guo et al., 2011). These experiments provided some new insights on the As mechanisms of release from Fe(oxyhydr-)oxides under in-stu conditions (Zhang et al., 2017)

We applied two surface complexation modeling approaches available in the geochemical code PHREEQC (i.e. DDL and CD-MUSIC) to simulate the desorption of As from goethite in order to provide quantitative interpretation of the in-situ experiments as well as to assess the performances of the 2 SCMs approaches under environmental multicomponent aqueous conditions.

\section{METHODS/EXPERIMENTAL}

A reactive transport model was developed in a Matlab ${ }^{\circledR}$ environment using the iPhreeqc module (Charlton \& Parkhurst, 2011). The transport of groundwater solutes and all geochemical reactions occurring at the surfaceaqueous interface were simulated through stepwise batch-type reactions occurring within single reactor cells. Such reactors represent the small containers encapsulating the As-loaded goethite-coated sand in the groundwater wells. The modeling implementation was based on data and results from the in-situ experiments performed in 7 wells (Zhang et al., 2017).

We assumed that multicomponent sorption competition was the only geochemical process leading to the release of As from goethite. This was hypothesized by (Zhang et al., 2017) according to the observed steady $\mathrm{Fe}$-content and absence of other $\mathrm{Fe}$-oxide phases rather than goethite in the in-situ experiments. Sorption reactions on goethite were modeled using the diffuse double layer approach (DDL) by Dzombak \& Morel (1990) and the charge distribution multisite complexation (CD MUSIC) model (Hiemstra \& Van Riemsdijk, 1996). The two distinct SCM approaches were applied in order to allow a critical comparison of the results and to assess the performances of the models when used for complex aqueous systems. For each SCM, a set of protonation/deprotonation and surface complexation reactions with their relative affinity constants $(\log K)$ was selected based on a thorough literature review.

The parameters (i.e. affinity constants, surface area, site densities, time step) were calibrated through a 
joint inversion procedure by minimizing the root mean squared error (RMSE) between predicted values and measured sorbed As onto goethite. Parameters calibration was performed (i) for each well individually and (ii) for the 7 wells simultaneously by parallelizing the simulation in order to find a unique set of parameters describing the experimental observations.

\section{RESULTS AND DISCUSSION}

A single set of parameters for both SCMs could provide satisfying fits of the measured adsorption As for all wells. However, simulation results showed significant differences applying the DDL and CD-MUSIC models respectively.

Figure 1 shows the result for a selected well and presents the temporal decrease of As from the surface of goethite predicted by the 2 SCMs where affinity constants were calibrated (CD-MUSIC 1 and DDL 1) or set equal to the best estimates reported in the literature. Without calibrating the affinity constants, the DDL model provides results closer to the measured data compared to the CD-MUSIC model. However, when calibration to the in-situ observations

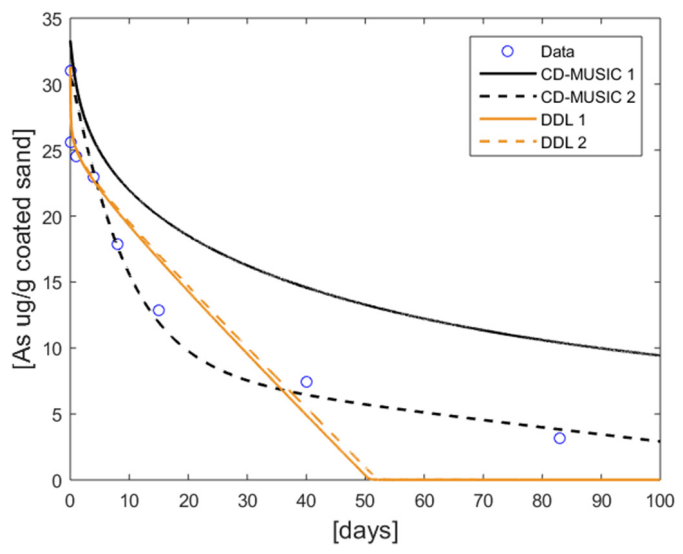

Figure 1. Temporal change in adsorbed As concentrations in a groundwater well. Dashed and full lines are results predicted by the SCMs. 1: parameters fixed to best estimates values re-ported in the literature. 2: parameters calibrated within range of values reported in the literature.
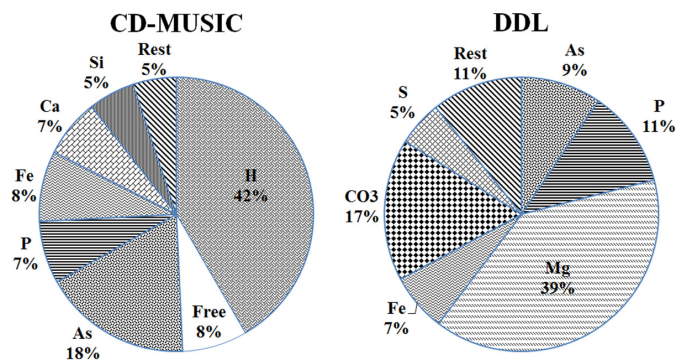

Figure 2. Surface composition at $\mathrm{t}=37$ days predicted by the CD-MUSIC 1 and DDL 1 model. was implemented, the CD-MUSIC allowed matching the measurements, whereas the performance of the DDL were not significantly improved (CD_MUSIC 2 and DDL 2).

The surface compositions predicted by the two SCMs were compared at times when the simulated adsorbed As was found equal between the DDL and the CD-MUSIC. Figure 2 shows that the two SCMs present significant difference in modeling the competition of the aqueous species for surface sites in the considered multicomponent aqueous system. The surface composition is dominated by protons in the CD-MUSIC model whereas magnesium is the dominant adsorbed specie in the DDL. The CD-MUSIC model does not predict any sorption of carbonate or sulfur unlike the DDL, but suggests sorption of silica and calcium.

\section{CONCLUSIONS}

Reactive transport modeling implementing surface complexation with the DDL and the CD-MUSIC models allowed us obtaining a reasonable agreement with the measured As-adsorbed concentrations in multicomponent aqueous conditions. However, the higher flexibility of the CD-MUSIC model provides a superior capability in fitting the experimental datasets with a unique set of parameters. Furthermore, the role of the aqueous species in the sorption competition significantly differs between the predictions of the DDL and the CD-MUSIC models.

\section{REFERENCES}

Charlton, S. \& Parkhurst, D. 2011. Modules based on the geochemical model PHREEQC for use in scripting and programming languages. Computat. Geosci. 37: 16531663.

Dzombak, D. \& Morel, F.M.M. 1990. Surface Complexation Modeling- Hydrous Ferric Oxide. New York: Wiley.

Guo, H., Zhang, B., Li, Y., Berner, Z., Tang, X., Norra, S. \& Stüben, D. 2011. Hydrogeological and biogeochemical constrains of arsenic mobilization in shallow aquifers from the Hetao basin, Inner Mongolia. Environ. Pollut. 159: 876-883.

Hiemstra, T. \& Van Riemsdijk, W. 1996. A surface structural approach to ion adsorption: the charge distribution (CD) model. J. Colloid Interf. Sci. 179: 488-508.

Neidhart, H., Berner, Z.A., Freikowski, D., Biswas, A., Majumder, S., Winter, J., Gallert, C., Chatterjee, D. \& Norra, S. 2014. Organic carbon induced mobilization of iron and manganese in a West Bengal aquifer and the muted response of groundwater arsenic concentrations. Chem. Geol. 367: 51-62.

Rawson, J., Prommer, H., Siade, A., Carr, J., Berg, M., Davis, J.A. \& Fendorf, S. 2016. Numerical modeling of arsenic mobility during reductive iron-mineral transformations. Environ. Sci. Technol. 50(5): 2459-2467.

Zhang, D., Guo, H., Xiu, W., Ni, P., Zheng, H. \& Wei, C. 2017. In-Situ Mobilization and Transformation of Iron OxidesAdsorbed Arsenate in Natural Groundwater. J. Hazard. Mater. 321: 228-237. 